\title{
Confiabilidad de la fuerza muscular respiratoria y flujos espiratorios forzados en adolescentes sanos
}

\author{
IVÁN RODRÍGUEZ N.*,*****
}

\section{Reliability of pulmonary function tests in healthy adolescents}

Inspiratory and expiratory muscle strength (MIP and MEP) assesment, and peak expiratory flow $(P E F)$ and peak cough flow (PCF) measurements are often used to assess patients with respiratory diseases. Notwithstanding, there are few reports about reliability of these measurements. The aim of this study is to determine the intra-observer reliability of MIP, MEP, PCF and PEF in healthy adolescents. The study design was test retest. A sample of students from a public school of Talcahuano (Chile) were studied. MIP, MEP, PEF and PCF were recorded twice by the same appraiser, with an interval of 8 weeks. Intraclass correlation coefficient (ICC) was used as an index of reliability. Analysis of individual differences was performed by Bland and Altman's method and the probability of discordance between test and retest was evaluated. A p value $<0.05$ was considered significant. 23 students (9 males), ageing $16.3 \pm 0.5$ years-old entered to the study. ICC of MIP, MEP, PEF and PCF were 0.7; $0.78 ; 0.86$ and 0.63 respectively. Bland-Altman analysis shows that the average discrepancy between the test and retest was -5 (95\% CI: 35.6 to -52.6), -9.1 (95\% CI: 20.2 to -38.4), -7.8 (95\% CI: 107.7 to -123.2) and -28.5 (95\% CI: 131.9 to -189.1) in MIP, MEP, PEF and PCF respectively. There was no significant difference in probability of discordance between parameters with the same unit of measure. Respiratory function variables show a moderate to high level of reliability, considering eight weeks of time interval between test and retest. PEF and MEP were the variables with the best performance.

Key words: Adolescents, reliability of measures, lung function.

\section{Resumen}

La evaluación de fuerza muscular inspiratoria (PImax) y espiratoria (PEmax), la medición del flujo espiratorio máximo (FEM) y flujo máximo de tos (FMT) son, a menudo, utilizados para el seguimiento de pacientes con enfermedades respiratorias. No obstante, son escasos los reportes acerca de sus propiedades de evaluación. El objetivo de este estudio es determinar la confiabilidad intraobservador de la PImax, PEmax, FMT y FEM, en adolescentes sanos. Se empleó un diseño test retest en que se seleccionaron adolescentes voluntarios de un colegio público de Talcahuano. PImax, PImax, FEM y FMT fueron registradas por un mismo evaluador en dos oportunidades con un intervalo de 8 semanas. Se empleó el coeficiente de correlación intraclase (CCI) como índice de confiabilidad. Mediante el método de Bland-Altman se realizó el análisis de las diferencias individuales y se evaluó la probabilidad de discordancia entre test y retest. Se consideró significativo un valor de $p<0,05$. Al estudio ingresaron 23 sujetos (9 varones), edad promedio 16,3 \pm 0,5 años. El CCI de PImax, PEmax, FEM y FMT fueron 0,7; 0,78; 0,86 y 0,63 respectivamente. Por su parte, el análisis de Bland-Altman muestra que el promedio de discordancia entre los tests fue -8,5 (95\% IC: 35, 6 a -52,6), -9, 1 (95\% IC: 20,2 a -38,4), -7,8 (95\% IC: 107,7 a -123,2) y-28,5 (95\% IC: 131,9 a -189,1) en PImax, PEmax,

* Docente, Escuela de Kinesiología, Facultad de Ciencias de la Salud, Universidad San Sebastián. Concepción, Chile.

** Kinesiólogo, Unidad de Ventilación Mecánica Prolongada, Servicio de Pediatría, Hospital Regional Dr. Guillermo Grant Benavente, Concepción, Chile.

*** Magíster en Fisiología Humana Universidad de Concepción; Alumno de Doctorado en Ciencias Médicas Universidad de la Frontera, Temuco, Chile - Programa de Pós-graduação em Farmacologia, Escola Paulista de Medicina, Universidade Federal de São Paulo, Brasil. 
FEM y FMT respectivamente. No existió diferencia significativa de probabilidad de discordancia entre parámetros con igual unidad de medida. Las variables de función respiratoria muestran un nivel de confiabilidad moderado a alto, considerando 8 semanas de intervalo de tiempo entre el test y retest. Las variables con mejor rendimiento fueron FEM y PEmax.

Palabras clave: Adolescentes, confiabilidad de las mediciones, función pulmonar.

\section{Introducción}

Diversas pruebas de función pulmonar son utilizadas para el diagnóstico y seguimiento de los pacientes con enfermedades respiratorias $(E R)^{1,2}$. En este contexto, la evaluación de los volúmenes y flujos forzados del sistema respiratorio mediante la espirometría constituye el método de evaluación de función pulmonar más ampliamente utilizado en los distintos niveles de atención de nuestro país ${ }^{3,4}$. Sin embargo, el costo de su implementación, así como también, el alto nivel de capacitación del personal técnico requerido para una apropiada ejecución ha permitido el surgimiento de métodos de evaluación simplificados para el seguimiento de los pacientes con enfermedades respiratorias ${ }^{3}$.

Es así como la medición del flujo espiratorio máximo (FEM) y flujo máximo de tos (FMT), a través del flujómetro mini-Wright ${ }^{\circledR}$, son variables frecuentemente medidas en el seguimiento de pacientes con enfermedades respiratorias ${ }^{5,6,7}$. Adicionalmente, la evaluación de la fuerza de los músculos inspiratorios (PImax) y espiratorios (PEmax) mediante la medición de las presiones generadas en la boca complementan las pruebas de función pulmonar, debido a que muestran una fuerte asociación con las capacidades pulmonares principalmente en pacientes neuromusculares ${ }^{8,9,10}$. En virtud de lo anterior, estas pruebas de función respiratoria son consideradas para el seguimiento del curso clínico de la enfermedad, así como también, como medida de efecto en la evaluación de alguna intervención con fines terapéuticos ${ }^{7,10}$.

Una de las principales desventajas de estas pruebas es que requieren de la motivación del paciente para una medición confiable. Lo cual, se encuentra sujeto al grado de aprendizaje de la técnica y experiencia del paciente en su ejecución ${ }^{1,6,8}$.

Diversos estudios que han evaluado las propiedades de estas pruebas, han comunicado rendimientos aceptables en cuanto a su confiabilidad $^{11,12,13}$. Se debe destacar que los intervalos de tiempo considerados entre el test y retest de los estudios citados se encuentran en el rango de días o semanas; no obstante, en el contexto clínico a menudo existe un intervalo de tiempo mayor debido al plan de seguimiento de la enfermedad o reevaluación posterior a alguna intervención ${ }^{3,7}$. Por este motivo, surge la necesidad de evaluar la confiabilidad de la medición de estos índices, considerando un intervalo temporal más amplio entre el test y re test, de manera similar a lo acontecido el contexto clínico.

Por lo precedentemente planteado el objetivo de este estudio es determinar la confiabilidad intraobservador del FEM, FMT y variables de fuerza muscular respiratoria, considerando 8 semanas de intervalo de tiempo entre las mediciones en una muestra de adolescentes chilenos sanos.

\section{Material y Método}

\section{Diseño de investigación}

En el presente estudio se empleó un diseño test retest con el fin de evaluar la confiabilidad intraobservador de las pruebas no invasivas de función respiratoria PImax, PEmax, FEM y FMT. Las pruebas fueron realizadas por el mismo evaluador con un intervalo de 8 semanas entre ellas.

\section{Muestra}

A través de un muestreo no probabilístico por conveniencia se seleccionó adolescentes voluntarios, sin restricción de género, estudiantes del colegio "Amanecer" de Talcahuano. Los criterios de inclusión fueron: jóvenes adolescentes sanos sedentarios (que realicen actividad física con una frecuencia menor a dos veces por semana). Como criterios de exclusión fueron considerados: la presencia de patología cardiorrespiratoria o músculoesquelética, sujetos que fumaban y aquellos que se encontraban bajo tratamiento broncodilatador. También se excluyó del estudio aquellos que presentaron algún cuadro de reagudización entre las evaluaciones.

\section{Variables}

En una planilla ad-hoc se registró, en ambas sesiones de evaluación, las variables biodemográficas (estatura, peso corporal, edad y género) y las variables de función respiratoria (PImax, PEmax, FEM y FMT). Para la medición del peso y talla se utilizó una cinta métrica y balanza análoga cuyo registro se expresó en centímetros $(\mathrm{cm})$ y kilogramos $(\mathrm{kg})$ respectivamente.

Tanto el FEM como el FMT fueron medidas 
con un flujómetro (Mini-Wright ${ }^{\circledR}$; Clement Clarke International, Essex, England). El FEM se midió con el sujeto en posición de pie, utilizando una pinza nasal, debiendo soplar con la mayor fuerza posible desde capacidad pulmonar total. La prueba se realizó un mínimo de 3 veces y un máximo de 8 ; se registró el valor más alto reproducible en 3 intentos con una diferencia no mayor al $10 \%$ entre cada prueba ${ }^{6}$. Por su parte, el FMT se midió con el sujeto sentado, lo cual favorece a la mecánica ventilatoria y permite un adecuado desarrollo de cada una de las fases de la tos. Durante la prueba se solicitó inspirar lo más profundo posible y realizar un esfuerzo de tos máximo a través del flujómetro. Los resultados fueron expresados en litros $/ \mathrm{min}^{7}$.

La fuerza de los músculos respiratorios se evaluó a través de la presión inspiratoria máxima (PImax), medida a través de un esfuerzo inspiratorio máximo mantenido por al menos un segundo desde volumen residual y la presión espiratoria máxima (PEmax) medida a través de un esfuerzo espiratorio máximo mantenido por al menos un segundo desde capacidad pulmonar total. Como instrumento se utilizó un manómetro aneroide NS120-TRS calibrado en centímetros de agua (desde 0 a -120 y desde $0 \mathrm{a}+120 \mathrm{cmH}_{2} \mathrm{O}$ ). Los valores obtenidos de PImax y PEmax se expresan en valores absolutos $\left(\mathrm{cmH}_{2} \mathrm{O}\right)^{7}$.

Finalmente, con el propósito de descartar algún cambio en la capacidad funcional de los músculos de la faja abdominal que pudiese influenciar el rendimiento de las pruebas de función respiratoria se evaluó la fuerza de los músculos abdominales (FMA) mediante el Sit Up Test; el cual, ha sido ampliamente utilizado, tanto en adolescentes sanos, como en pacientes con enfermedades respiratorias crónicas ${ }^{14,15}$. El protocolo de ejecución se llevó a cabo de acuerdo a lo publicado por nuestro grupo ${ }^{14}$.

\section{Consideraciones éticas}

Ningún integrante de la muestra participó en otros estudios realizados previamente ${ }^{14}$. Adicionalmente, se contó con la autorización del establecimiento educacional para la realización de este estudio y todos los padres firmaron un consentimiento y los adolescentes un asentimiento informado.

\section{Análisis estadístico}

Utilizando el paquete estadísticos MedCalc 14,8; se realizó un análisis exploratorio de los datos y posteriormente se aplicó estadística descriptiva con cálculo de promedio y desviación estándar (DS). Se utilizó t-student para muestras pareadas con el fin de evaluar la diferencia de las variables entre el primer y segundo test (muestra total), así como también, fue utilizado el test $U$ Mann Whitney para evaluar las variables entre géneros y el test de Wlcoxon para evaluar la magnitud de las diferencias entre géneros entre el pre y post. Por otra parte, se empleó el coeficiente de correlación intraclase (CCI) para evaluar la confiabilidad de las variables de función respiratoria. Adicionalmente, mediante el método de Bland-Altman, se realizó el análisis de las diferencias individuales mediante la relación entre el promedio de la diferencia entre ambos tests y el límite de concordancia. Finalmente, se evaluó la probabilidad de discordancia entre el test y retest. El test log rank fue utilizado para comparar la probabilidad de discordancia entre variables de iguales unidades de medida (PImax-PEmax y FEM-FMT). Se consideró significativo un valor de $\mathrm{p}<0,05$.

\section{Resultados}

\section{Caracterización de la muestra}

$\mathrm{Al}$ estudio ingresaron 23 sujetos (9 varones), edad promedio $16,3 \pm 0,5$ años. Ningún sujeto fue excluido durante el desarrollo del estudio debido a presencia de algún cuadro de reagudización. No existió diferencia en las variables biodemográficas ni en la fuerza de los músculos abdominales medida por el Sit Up Test entre el test y retest (Tabla 1).

\section{Resultados descriptivos de las pruebas de función respiratoria}

En los hombres se observaron mayores valores de PEmax, FEM, FMT y FMA respecto a las mujeres tanto en el pre como en el post test. La magnitud de la diferencia entre géneros fue semejante tanto en el test como el retest (Tabla 2). Adicionalmente, en todas las pruebas se observaron mayores valores absolutos en el retest, sin embargo, sólo la variable PEmax incrementó de manera estadísticamente significativa $(\mathrm{p}=0,007)$ (Tabla 3).

\section{Análisis de confiabilidad}

El coeficiente de correlación intraclase (CCI) calculado para cada una de las pruebas de función respiratoria fue de moderado a fuerte (Tabla 4). Por otra parte, el análisis de Bland-Altman muestra que el promedio de discordancia entre los tests fue $-8,5(35,6$ a $-52,6),-9,1(20,2$ a $-38,4)$, $-7,8(107,7$ a $-123,2)$ y $-28,5(131,9$ a $-189,1)$ en PImax, PEmax, FEM y FMT respectivamente (Figuras 1 a 4 ). 
Por otra parte, no se observó diferencia significativa en la probabilidad de discordancia entre las pruebas de fuerza muscular respiratoria (PImax y PEmax), ni flujos espiratorios forzados (FEM y FMT) (Figuras 5 y 6 ).

Tabla 1. Características generales de los 23 adolescentes sedentarios del estudio

\begin{tabular}{|lcc|}
\hline Variables & Test & Retest \\
\hline Género $(\mathrm{H} / \mathrm{M})$ & $9 / 14$ & $9 / 14$ \\
\hline Edad (años) & $16,3 \pm 0,5$ & $16,3 \pm 0,5$ \\
Peso $(\mathrm{kg})$ & $61,6 \pm 11,0$ & $61,8 \pm 10,8$ \\
Talla $(\mathrm{cm})$ & $162,9 \pm 8,3$ & $162,9 \pm 8,3$ \\
Sit up test & $23,3 \pm 6,7$ & $23,4 \pm 7,2$ \\
\hline
\end{tabular}

Resultados se muestran en promedio \pm su desviación estándar. No hubo diferencias significativas entre el test y retest en ninguna de las variables (se usó t-test para muestras pareadas).
Tabla 2. Diferencias en el resultado de las pruebas de función respiratoria y fuerza de músculos abdominales de acuerdo al género

\begin{tabular}{|lcc|}
\hline Variable & Test & Retest \\
\hline$\Delta$ género PImax, $\%$ & $7,0^{\mathrm{ns}}$ & $9,0^{\mathrm{ns}}$ \\
\hline$\Delta$ género PEmax, $\%$ & $33,3^{*}$ & $19,0^{*}$ \\
\hline$\Delta$ género FEM, $\%$ & $42,4^{* * *}$ & $17,5^{* *}$ \\
\hline$\Delta$ género FMT, $\%$ & $23,5^{* *}$ & $11,3^{* *}$ \\
\hline$\Delta$ género Sit up test, $\%$ & $57,8^{* * *}$ & $40^{* *}$ \\
\hline
\end{tabular}

PImax: Presión inspiratoria máxima; PEmax: Presión espiratoria máxima; FEM: Flujo espiratorio máximo; FMT: Flujo máximo de tos. $\Delta$ género fue calculada como: (Variable hombre-Variable mujer) / Variable mujer x 100. ns: no significativo; $*<0,05 ; * *<0,01 ; * * *<0,001$. (Test U de Mann Whitney). - No existió diferencia significativa entre los test respecto a las diferencia según género (Test de Wilcoxon $\mathrm{p}=0,1)$.
Tabla 3. Resultados de las pruebas de función respiratoria en el test y retest de la muestra total

\begin{tabular}{|lcccl|}
\hline Variables & Test & Retest & \% $\Delta$ & \multicolumn{1}{c|}{$\mathbf{p}^{*}$} \\
PImax $\left(\mathrm{cmH}_{2} \mathrm{O}\right)$ & $98,2 \pm 24,4$ & $108,1 \pm 16,9$ & $+10,2$ & 0,08 \\
PEmax $\left(\mathrm{cmH}_{2} \mathrm{O}\right)$ & $78,1 \pm 19,0$ & $93,6 \pm 16,2$ & $+19,8$ & 0,007 \\
FEM $(\mathrm{L} / \mathrm{min})$ & $416,6 \pm 95,7$ & $424,3 \pm 76,1$ & $+1,8$ & 0,5 \\
FMT $(\mathrm{L} / \mathrm{min})$ & $382,2 \pm 89,1$ & $410,8 \pm 66,1$ & $+7,5$ & 0,10 \\
\hline
\end{tabular}

$\% \Delta$ : Porcentaje de cambio, calculado como: valor retest-valor test/ valor test; PImax: Presión inspiratoria máxima; PEmax: Presión espiratoria máxima; FEM: Flujo espiratorio máximo; FMT: Flujo máximo de tos. Resultados se muestran en promedio \pm su desviación estándar. *t-test para muestras pareadas.
Tabla 4. Coeficiente de correlación intraclase de las variables de función respiratoria

\begin{tabular}{|cc|}
\hline Variables & CCI \\
\hline PImax $\left(\mathrm{cmH}_{2} \mathrm{O}\right)$ & 0,70 \\
PEmax $\left(\mathrm{cmH}_{2} \mathrm{O}\right)$ & 0,78 \\
\hline FEM $(\mathrm{L} / \mathrm{min})$ & 0,86 \\
FMT $(\mathrm{L} / \mathrm{min})$ & 0,63 \\
\hline
\end{tabular}

PImax: Presión inspiratoria máxima; PEmax: Presión espiratoria máxima; FEM: Flujo espiratorio máximo; FMT: Flujo máximo de tos; CCI: Coeficiente de correlación intraclase.

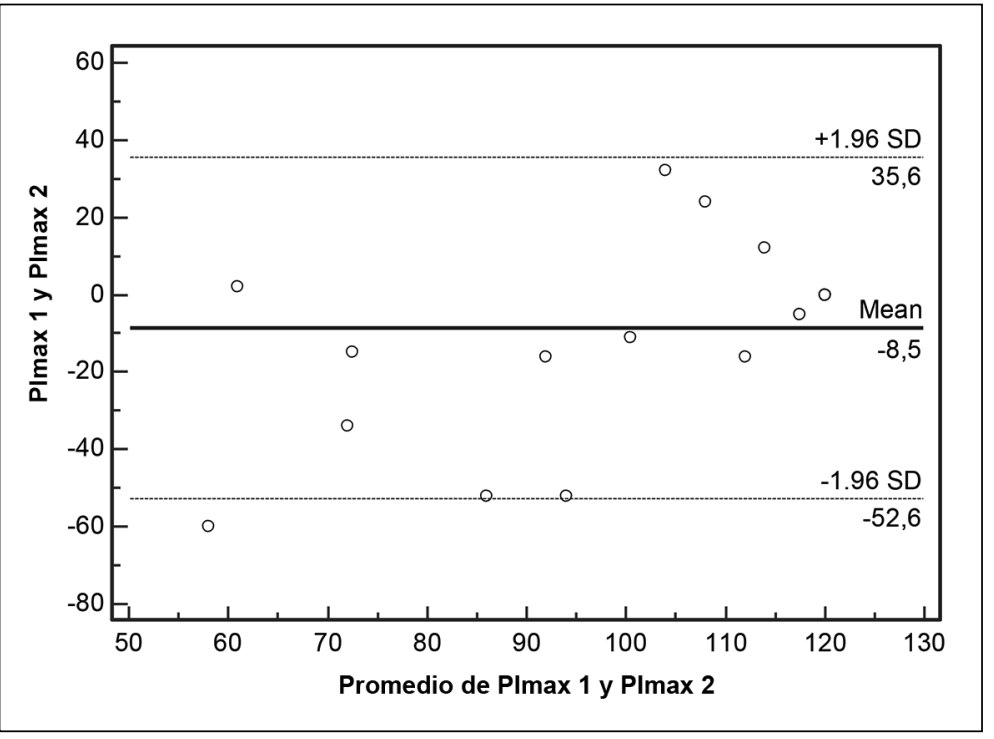

Figura 1. Confiabilidad test retest de la presión inspiratoria máxima (PImax). Resultados se muestran en promedio e intervalo de confianza. 

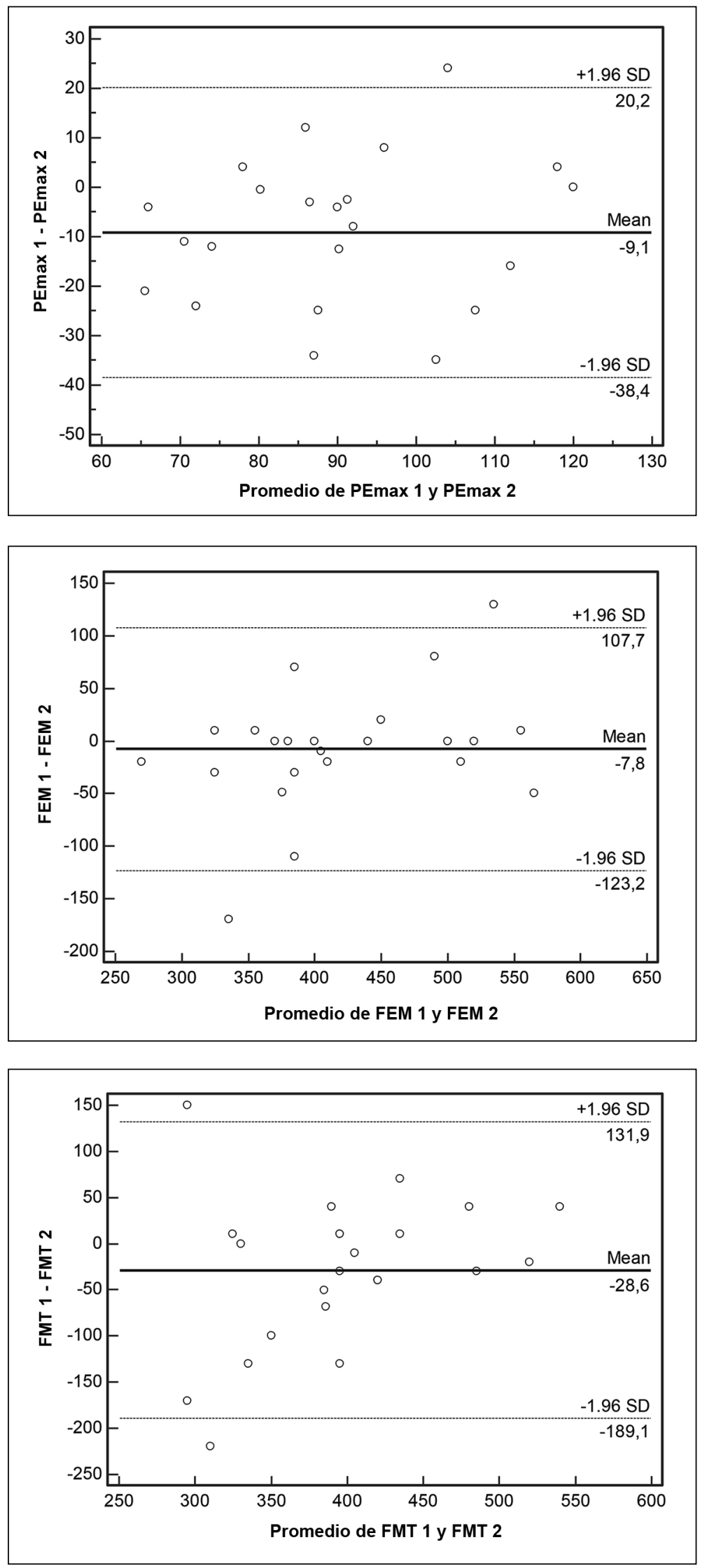

Figura 2. Confiabilidad test retest de la presión espiratoria máxima (PEmax). Resultados se muestran en promedio e intervalo de confianza.

Figura 3. Confiabilidad test retest del flujo espiratorio máximo (FEM). Resultados se muestran en promedio e intervalo de confianza.

Figura 4. Confiabilidad test retest del flujo máximo de tos (FMT). Resultados se muestran en promedio e intervalo de confianza. 

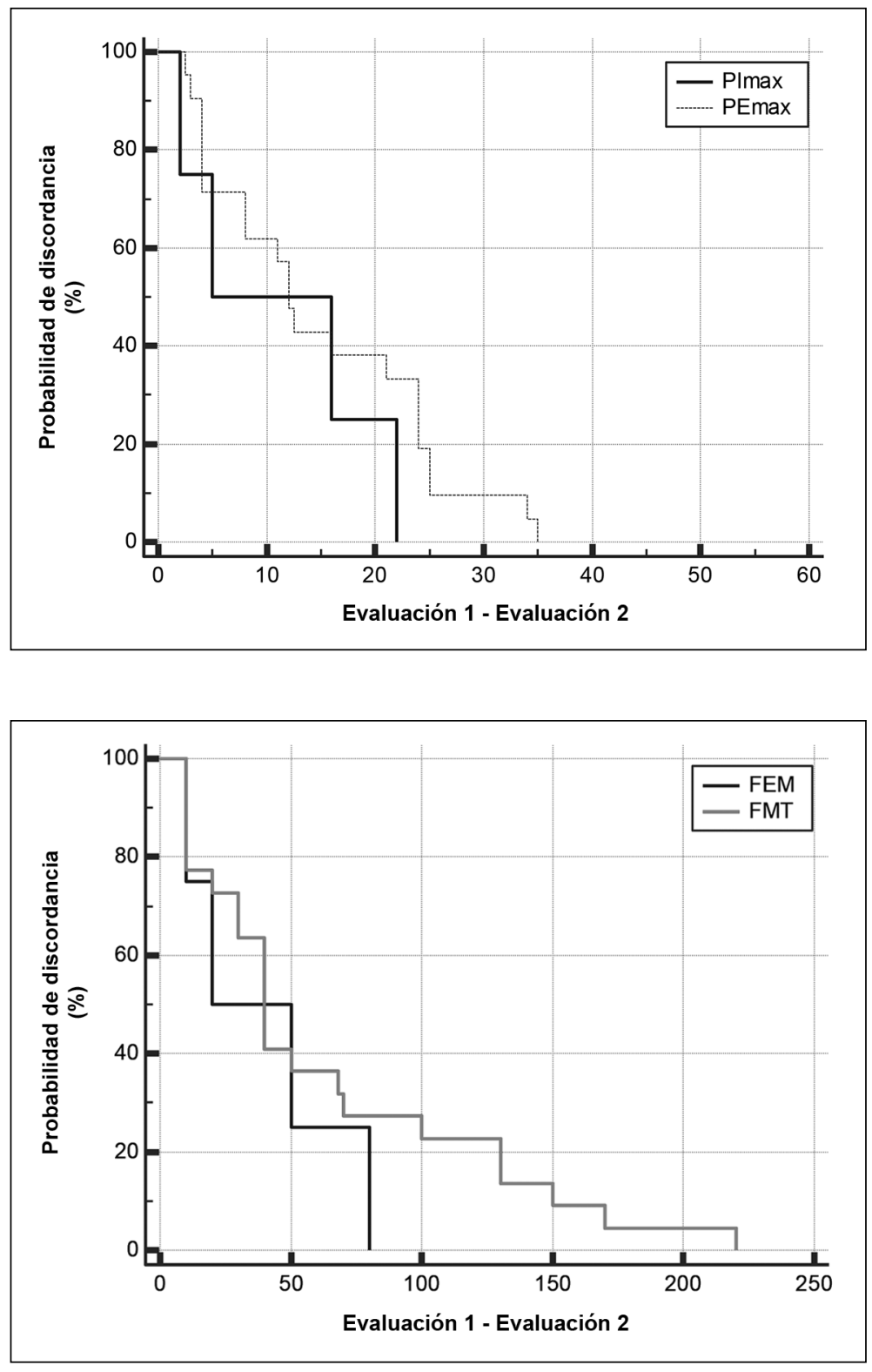

Figura 5. Probabilidad de discordancia entre el test y retest de PImax y PEmax; $\mathrm{p}=0,25$ (Log rank test $)$.
Figura 6. Probabilidad de discordancia entre el test y retest de FEM y FMT. $\mathrm{p}=0,30($ Log rank test $)$.

\section{Discusión}

En el presente estudio fue verificada la confiabilidad intra-observador de las variables de función respiratoria más ampliamente medidas en el seguimiento de pacientes con enfermedades respiratorias. Los resultados muestran que las variables poseen diversos niveles de confiabilidad, siendo FMT y FEM, las variables que mostraron los más bajos y los más altos índices de confiabilidad (CCI: 0,63 y 0,86 respectivamente).

Como índice de confiabilidad inter-observador fue considerado el coeficiente de correlación intraclase $(\mathrm{CCI})$, el cual, representa la proporción de la variabilidad total que no es producida por error de medición ${ }^{16}$. Adicionalmente, como variables de control fueron consideradas las características biodemográficas de los sujetos (peso, talla, edad), así como también, el rendimiento durante el Sit up test observándose en ninguna de estas variables diferencias significativas entre el test y el retest. Esto permite descartar la presencia de error sistemático asociado a cambios antropométricos o fisiológicos de los individuos que pudiesen afectar el nivel de confiabilidad obtenido.

Son escasos los estudios donde se ha evaluado el nivel de confiabilidad en este tipo de variables. Morán y cols ${ }^{12}$, verificaron la confiabilidad de la PImax y PEmax en 20 sujetos con bronquiectasias no asociada a fibrosis quística, observando 
un alto nivel de confiabilidad en ambas mediciones. Cabe destacar que el intervalo de tiempo considerado fue de sólo un día, pese a aquello, fueron observadas diferencias absolutas en los valores medidos entre los tests, siendo estadísticamente significativo sólo en la variable PEmax $\left(+10 \mathrm{cmH}_{2} \mathrm{O} ; \mathrm{p}=0,02\right)$.

Los resultados del estudio citado concuerdan con lo observado en el presente estudio donde también existió diferencia significativa sólo en la PEmax $\left(+19 \mathrm{cmH}_{2} \mathrm{O} ; \mathrm{p}=0,007\right)$. Pese a lo anterior, el rendimiento del Sit up test fue similar en ambas pruebas, lo cual sugiere que el desarrollo de la fuerza de la faja abdominal para ejecutar la espiración forzada no se asocia al requerido para ejecutar el Sit up test. Esto concuerda con lo recientemente reportado por nuestro grupo donde fue observada la inexistencia de correlación entre la FMA y PEmax ${ }^{14}$. Por otra parte, un potencial fenómeno de aprendizaje en el desarrollo de las pruebas de evaluación de la fuerza muscular respiratoria durante retest no puede ser descartado debido a la magnitud de las diferencias observadas entre los test tanto en la PImax como en la PEmax.

En relación a la medición de flujos forzados la variable FEM, pese a existir diferencias absolutas entre el test y retest, mostró el nivel de confiabilidad más fuerte observado (CCI: 0,86). Esto concuerda con lo reportado por Mandros y cols ${ }^{17}$, que observó un alto nivel de reproducibilidad en la medición del FEM, considerando diversos tipos de maniobras estandarizadas. Por su parte, Holcroft y cols ${ }^{11}$, observaron un bajo porcentaje de variación entre 5 mediciones consecutivas de FEM en una muestra de 223 sujetos sanos, siendo las variaciones asociadas a hiperreactividad bronquial, hipersecreción y hábito tabáquico. No obstante, las mediciones fueron realizadas en días consecutivos durante una semana; diferenciándose de nuestro protocolo, el cual se llevó a cabo en un intervalo de 2 meses.

El FMT mostró el nivel más bajo de confiabilidad. En este ámbito, se debe considerar que el desarrollo de los flujos espiratorios durante la tos demanda un nivel de aprendizaje específico para un adecuado desarrollo de cada una de las fases que la componen ${ }^{18}$. En virtud de lo anterior, la maniobra de la tos presentaría una mayor fuente de variabilidad que las otras pruebas de función respiratoria, lo cual fue observado en este estudio al presentar un $37 \%$ de error sistemático de medición.

Mediante el análisis de probabilidad de discordancia $^{19}$ fue posible constatar que no existe diferencia entre la variable PImax y PEmax, así como también, entre la variable FEM y FMT en cuanto al riesgo de presentar discordancia en mediciones sucesivas. Lo cual muestra que las pruebas función pulmonar con magnitudes físicas semejantes comparten ciertas propiedades de medición a pesar de ser ejecutadas de manera diferente.

Vale la pena destacar que se observó un mayor nivel de PEmax, FEM, FMT y FMA en sujetos de género masculino, tanto en el pre como post test (Tabla 2). A pesar que en el post test se observó una disminución en la magnitud de estas diferencias, esta no fue estadísticamente significativa (test de Wilcoxon $\mathrm{p}=0,1$ ). Por tanto, es probable que la variabilidad existente entre hombres y mujeres, tanto en el pre como en el post test, no haya afectado significativamente los niveles de confiabilidad observados en este estudio. No obstante, dado el pequeño tamaño muestral considerado no es posible descartar esta posible fuente de sesgo.

A partir de los resultados de este estudio, es posible conjeturar que la magnitud del efecto inducido por intervenciones terapéuticas como el entrenamiento muscular respiratorio (EMR) debe ser analizada con cautela, debido a que la falta de confiabilidad puede conducir a errores de sobreestimación de los resultados obtenidos posterior a la intervención. Resultados recientemente publicados por nuestro grupo han mostrado que 8 semanas de entrenamiento de músculos abdominales incrementaron la PImax, PEmax 17,1\% y $50,3 \%$ respectivamente en adolescentes sanos ${ }^{14}$. Adicionalmente, estudios previos en pacientes con enfermedad respiratoria crónica ya habían mostrado que 13 y 24 semanas de entrenamiento de los músculos respiratorios generan incrementos en la fuerza muscular respiratoria superiores a $15 \%$ y $30 \%$ respectivamente ${ }^{7,20}$. Estas diferencias son superiores a las inducidas por el error aleatorio observadas en los resultados de este estudio, lo cual podría sugerir que las variables de función respiratoria analizadas serían capaces de reconocer los cambios en el estatus funcional de una persona que es sometida a este tipo de entrenamiento.

A partir de este reporte es posible formular nuevas hipótesis que deben ser verificadas en estudios ulteriores. En este sentido, evaluar el efecto de distintos intervalos de tiempo entre mediciones sucesivas, así como también, el impacto de distintos protocolos de educación al paciente sobre las propiedades de medición permitiría reorientar los planes y programas de seguimiento de los pacientes con enfermedades respiratorias, con el fin de realizar mediciones con más altos índices de confiabilidad que favorezcan la precisión de los datos obtenidos. 
Finalmente, los resultados de este estudio sugieren que las variables de función respiratoria muestran diversos niveles de confiabilidad. En este contexto, el FMT y FEM fueron las variables que mostraron los más bajos y más altos índices de confiabilidad respectivamente. Futuros estudios con mayor tamaño muestral son necesarios para confirmar estas conclusiones.

\section{Bibliografía}

1.- BEYDON N, DAVIS S D, LOMBARDI E, ALLEN J L, ARETS H G, AURORA P, et al. An official American Thoracic Society/European Respiratory Society statement: pulmonary function testing in preschool children. Am J Respir Crit Care Med 2007; 175: 1304-45.

2.- NARDINI S, ANNESI-MAESANO I, DEL DONNO M, DELUCCHI M, BETTONCELLI G, LAMBERTI V, et al. The AIMAR recommendations for early diagnosis of chronic obstructive respiratory disease based on the WHO/GARD model*. Multidiscip Respir Med 2014; 9 : 46.

3.- Ministerio de Salud. Guía Clínica Asma bronquial moderada y grave en menores de 15 años. Minsal, 2011.

4.- Ministerio de Salud. Guía Clínica Enfermedad Pulmonar Obstructiva Crónica de tratamiento ambulatorio. Santiago: Minsal, Santiago. 2013.

5.- SEPÚLVEDA R. El flujómetro de Wright. Una herramienta indispensable en la práctica ambulatoria. Rev Chil Enferm Respir 2004; 20: 80-4.

6.- CÉSPEDES J, GUTIÉRREZ M, OYARZÚN M. Flujometría en la práctica de atención primaria. Rev Chil Enferm Respir 2010; 26: 47-8.

7.- RODRÍGUEZ I, ZENTENO D, MANTEROLA C. Effects of home-based respiratory muscle training in children and adolescents with chronic lung disease. J Bras Pneumol 2014; 40: 626-33.

8.- FAUROUX B, QUIJANO-ROY S, DESGUERRE I, KHIRANI $S$. The value of respiratory muscle testing in children with neuromuscular disease. Chest 2015; 147: 552-9.

9.- FAUROUX B, AUBERTIN G, CLÉMENT A, LOFASO F, BONORA M. Which tests may predict the need for noninvasive ventilation in children with neuromuscular disease? Respir Med 2009; 103: 574-81.

10.- NICOT F, HART N, FORIN V, BOULÉ M, CLÉMENT A, POLKEY M I, et al. Respiratory muscle testing: a valuable tool for children with neuromuscular disorders. Am J Respir Crit Care Med 2006; 174: 67-74.

11.- HOLCROFT C A, EISEN E A, SAMA S R, WEGMAN D H. Measurement characteristics of peak expiratory flow. Chest 2003; 124: 501-10.

12.- MORAN F, PIPER A, ELBORN J S, BRADLEY J M. Respiratory muscle pressures in non-CF bronchiectasis: repeatability and reliability. Chron Respir Dis 2010; 7 : 165-71.

13.- ROMER L M, MCCONNELL A K. Inter-test reliability for non-invasive measures of respiratory muscle function in healthy humans. Eur J Appl Physiol 2004; 91: 167-76.

14.- RODRÍGUEZ I, ALARCÓN M, GUTIÉRREZ C, HERMOSILLA P, CONTRERAS T, BÁEZ C. Efecto del entrenamiento de músculos abdominales sobre la función respiratoria en adolescentes sanos. Estudio piloto. Rev Chil Enferm Respir 2014; 30: 203-11.

15.- ELBASAN B, TUNALI N, DUZGUN I, OZCELIK U. Effects of chest physiotherapy and aerobic exercise training on physical fitness in young children with cystic fibrosis. Ital J Pediatr 2012; 38: 2.

16.- ZOU G Y. Sample size formulas for estimating intraclass correlation coefficients with precision and assurance. Stat Med 2012; 31: 3972-81.

17.- MANDROS C, TSIAKALOS A, TZELEPIS G E. Intersession reproducibility of peak expiratory flow with standardized expiratory maneuvers. Respir Med 2007; 101: 933-7.

18.- MCCOOL F D. Global physiology and pathophysiology of cough: ACCP evidence-based clinical practice guidelines. Chest 2006; 129 (1 Suppl): 48S-53S.

19.- LUIZ R R, COSTA A J, KALE P L, WERNECK G L. Assessment of agreement of a quantitative variable: a new graphical approach. J Clin Epidemiol 2003; 56 : 963-7.

20.- RODRÍGUEZ I, FUENTES C, RIVAS C, MOLINA F, SEPÚLVEDA C, ZENTENO D. Rehabilitación respiratoria en el paciente neuromuscular: efectos sobre la tolerancia al ejercicio y musculatura respiratoria. Resultado de una serie de casos. Rev Chil Enferm Respir 2013; 29 196-203.

Correspondencia a:

Klgo. Iván Rodríguez Núñez

Universidad San Sebastián, Facultad de Ciencias de la

Salud, Centro de Medicina Molecular. Lientur 1457.

Concepción, Chile.

Email: ivan.rodriguez@uss.cl 